\title{
P4HA2 Gene
}

National Cancer Institute

\section{Source}

National Cancer Institute. P4HA2 Gene. NCI Thesaurus. Code C101549.

This gene is involved in collagen metabolism. 